\title{
Ferramenta virtual como método complementar no ensino da anatomia na espécie felina
}

\author{
Virtual tool as a complementary method on the anatomy learning \\ process of feline species
}

\author{
Dala Kezen Vieira Hardman Leite (iD ${ }^{1 *}$, Nathália Ferreira Corrêa (iD) ${ }^{1}$, Thais Cristina Belondre Andrade (iD ${ }^{1}$, Milene \\ Couras da Cruz (D)², Rômulo Ferreira de Assumção (D) ${ }^{1}$ \\ 1 Universidade Iguaçu (UNIG) , Nova Iguaçu, RJ, Brasil \\ 2 Pontificia Universidade Católica do Rio de Janeiro (PUC-Rio), Rio de Janeiro, RJ, Brasil
}

\section{Resumo}

A fim de verificar o impacto positivo da ferramenta virtual como método complementar no ensino de anatomia, conforme abordado em diversas pesquisas recentes, o presente trabalho realizou um estudo primário sobre imagens anatômicas topográficas virtuais 3D nos sistemas digestório, urinário, respiratório e cardiovascular na espécie felina. Os docentes e discentes participaram do presente estudo, tendo sido treinados e capacitados para utilização do software (5.02) da Mesa Anatômica Virtual 3D (Anatomage). No sistema digestório foram identificados: esôfago, estômago, intestino delgado e intestino grosso; no entanto, no software não foi possível detalhar a cavidade oral. Em relação ao sistema urinário, as estruturas como rins, ureteres, vesícula urinária e uretra foram identificadas. Com referência ao sistema respiratório, os pulmões, laringe e a traqueia foram estruturas evidenciadas. Contudo, os brônquios, porção nasal da laringe e cavidade nasal não puderam ser exploradas, carecendo de detalhamento do software. Por fim, no sistema cardiovascular, as estruturas do coração foram identificadas e dissecadas, apesar de a vascularização não ter sido visualizada. Após analisar os sistemas, observar cada órgão espacialmente e identificar as estruturas, a dissecção virtual foi empregada para investigação anatômica mais detalhada. Durante o processo foram produzidas imagens anatômicas em diversos planos (rostral, ventral, lateral e sagital). Os recursos presentes no software certificaram que a mesa anatômica virtual 3D é uma ferramenta facilitadora, interativa e dinâmica. Posterior à capacitação da equipe, os sistemas estudados na espécie felina foram

* Autor correspondente: dkezen@gmail.com 
explorados, identificados e dissecados de forma clara e simples. Uma atualização da variabilidade das espécies animais no software beneficiará o ensino aprendizagem da anatomia veterinária. A mesa anatômica virtual 3D apresenta um potencial que contribuirá de forma positiva na metodologia ativa de forma a complementar o ensino tradicional no estudo da anatomia.

Palavras-chave: Anatomia veterinária. Espécie felina. Mesa anatômica virtual 3D.

\section{Abstract}

In order to verify the positive impact of the virtual tool as a complementary method on the anatomy learning process, as adressed in several current research, the present work conducted an initial virtual 3D topographic anatomical study on the digestive, urinary, respiratory, and cardiovascular systems of the feline species. Professors and students were trained and qualified to use the software (5.02) 3D Virtual Anatomical Table (Anatomage). Regarding the digestive system, components as esophagus, stomach, small intestine and large intestine were identified; however, it was impossible to study the oral cavity in detail. As for the urinary system, structures such as kidneys, ureters, urinary bladder and urethra were also identified. With regard to the respiratory system, structures as lungs, larynx and trachea were evidenced but, unfortunately, bronchi, nasal portion of the larynx and nasal cavity could not be explored in behalf of the software's lack of details. At last, about the cardiovascular system, the structures of the heart were identified and dissected, although vascularization could not be visualized. After analyzing the systems, observing spaces of each organ and identifying structures, a virtual dissection was employed in each framework for a more detailed anatomical investigation. During the process, anatomical images were developed in several planes (rostral, ventral, lateral, sagittal). The features in the software have certified that the $3 D$ virtual anatomical table is an useful, interactive and dynamic tool. After training the team, the studied feline species' systems were explored, identified and dissected in a clear and simple way. An update on the software's animal species variability would greatly benefit the teaching and learning of veterinary anatomy. The 3D virtual anatomical table has a strong potential to contribute to the active learning method as a complementary tool to the traditional education system applied on the anatomy study.

Keywords: Veterinary anatomy. Feline species. 3D virtual anatomical table.

\section{Introdução}

O ensino tradicional da anatomia é feito por meio de aulas expositivas teóricas e práticas, desenvolvidas em laboratórios com exposição de peças anatômicas dissecadas para estudo das estruturas (Cintra, 2017). Reis et al. (2013) afirmam que os alunos não se sentem motivados para aprender terminologia anatômica e defendem que um método mais participativo ajudaria a melhorar a motivação e o aprendizado. Salbego et al. (2015) reiteram que o docente deve utilizar práticas inovadoras e conhecer propostas alternativas para estimular a participação do discente. Diante das circunstâncias, o ensino da anatomia humana e veterinária deve ser debatido para corresponder às expectativas deste atual momento (Custer e Michael, 2015; Rinaldi et al., 2017; Chung et al., 2018; Corrêa et al., 2019).

Os acadêmicos enfrentam outras dificuldades perante a metodologia das aulas práticas, tais como peças anatômicas inadequadas, escassez de cadáveres, problemas alérgicos e receio do manuseio de cadáveres (Reis et al., 2013). Lisk et al. (2015), Raffan et al. (2017) e Schoenfeld-Tacher et al. (2017) sinalizam que a região anatômica estudada também é um fator limitante relevante em aulas práticas, pois na dissecção de um cadáver formolizado não é possível explorar de forma adequada regiões com superfícies muito reduzidas. Ademais, Karam et al. (2016) comprovaram que a utilização de formol em peças anatômicas pode gerar consequências danosas à saúde de estudantes, profissionais de anatomia e ao meio ambiente.

Para o estudo da anatomia humana e animal, temos encontrado diversas técnicas além da formolização,comoglicerinização,criodesidratação e plastinação. A técnica de glicerinação se 
apresentou como uma alternativa para o uso do formol por ser inodora, garantir coloração da peça anatômica mais próxima da realidade e gerar maior leveza (Santos et al., 2017a; Fontoura et al., 2020). A criodesidratação é implementada mediante congelamento e descongelamento repetitivos de peças anatômicas para extração de toda a água do material. É um método que dispensa soluções conservantes, porém os tecidos retraem e as peças se tornam rígidas e frágeis, suscetíveis a rompimento durante o manuseio. A plastinação é uma técnica executada em quatro etapas: fixação, desidratação, impregnação e secagem. A técnica apresenta alta resistência e durabilidade ao material anatômico, mantendo as características originais da peça; todavia, é um método altamente dispendioso e de difícil realização (Fontoura et al., 2020).

O uso de cadáveres formolizados ainda é imprescindível para o entendimento da anatomia e, por esta razão, não é uma metodologia substituível (Gummery et al., 2018; Massari et al., 2018; Silva et al., 2018). Entretanto, diferente do método tridimensional virtual, a constante manipulação das peças formolizadas pode danificar as estruturas mais sensíveis, prejudicando as pesquisas (Fazan, 2011).

O impacto positivo da ferramenta virtual como método complementar no ensino de anatomia vem sendo salientado em diversas pesquisas recentes. Os efeitos da integração da Mesa Anatômica Virtual 3D (Anatomage) são percebidos em diversos âmbitos. Os benefícios em relação ao ensino-aprendizagem englobam: dinamismo em aula, metodologia ativa, aumento da compreensão de assuntos, diminuição de contato com formol, recurso renovável e possibilidade do discente conduzir o seu estudo de acordo com seu próprio ritmo de aprendizagem (Brown, et al., 2015; Moraes et al., 2016; Santos et al., 2017b; Massari, et al., 2018; Corrêa et al., 2019; Little et al., 2019; Periya e Moro, 2019).

A utilização de tecnologias de ensinoaprendizagem em animais ainda não é frequente, entretanto, algumas empresas já começaram a desenvolver softwares que demonstraram ser de grande utilidade para a medicina humana e veterinária, eliminando a subjetividade e contornando os obstáculos dos métodos convencionais (Gross e Masters, 2017; Fyfe et al., 2018; Corrêa et al., 2019). Autores como Lisk et al. (2015), Azer e Azer (2016), Raffan et al. (2017), Schoenfeld-Tacher et al. (2017) e Corrêa et al. (2019) concordam que diversos elementos como design do software 3D, disponibilidade visual da peça, interação cognitiva no uso do software e região anatômica são princípios significativos e fundamentais para o ensino-aprendizagem. Portanto outra metodologia de ensino da anatomia vem sendo estudada e utilizada como uma forma alternativa e complementar, frisando cada vez mais a magnitude e prevalência da tecnologia na educação (Reis et al., 2017; Lima et al., 2018).

A anatomia animal é de grande relevância no curso de medicina veterinária. Os sistemas orgânicos digestório, urinário, respiratório e cardiovascular são de extrema importância no currículo do discente, ressaltando o conhecimento e habilidade no ensino prático.

O presente trabalho realizou um estudo primário sobre imagens anatômicas topográficas virtuais 3D nos sistemas digestório, urinário, respiratório e cardiovascular na espécie felina contidas no software (5.02) da Mesa Anatômica Virtual 3D (Anatomage).

\section{Material e métodos}

Dois docentes e quatro discentes participaram do presente estudo, tendo sido treinados e capacitados para utilização do software (5.02) da Mesa Anatômica Virtual 3D (Anatomage Interactive Anatomy Study Table) no estudo anatômico e topográfico virtual da espécie felina. A pesquisa foi realizada no Laboratório Anatômico do Departamento de Medicina da Pontifícia Universidade Católica do Rio de Janeiro (PUC-Rio), no ano de 2019. O treinamento e capacitação duraram em torno de seis meses, durante os quais foram apresentadas as funções e as ferramentas da mesa, permitindo que os envolvidos explorassem todos os arquivos disponíveis a referentes animais.

Os sistemas digestório, urinário, respiratório e cardiovascular da espécie felina foram explorados, 
visualizados, identificados e dissecados em diversos planos. As estruturas anatômicas virtuais exploradas no sistema digestório foram: boca, faringe, esôfago, estômago, intestino delgado e intestino grosso; no sistema urinário: rins, ureteres, vesícula urinária e uretra; no sistema respiratório: cavidade nasal, porção nasal da faringe, laringe, traqueia, brônquios e pulmões. No sistema cardiovascular, as estruturas do coração foram identificadas e dissecadas, entretanto, a vascularização não pôde ser visualizada, pois o software carece do detalhamento vascular nas peças animais.

Os procedimentos aplicados durante o estudo virtual foram semelhantes às etapas realizadas no estudo com cadáveres formolizados. Após estudo dos sistemas, observação espacial de cada órgão e identificação das estruturas, a dissecção virtual foi empregada em cada estrutura para investigação anatômica mais detalhada.

Das diversas ferramentas disponibilizadas pelo software da mesa anatômica Anatomage, algumas foram muito utilizadas, pois norteavam/ direcionavam as seleções das partes a serem estudadas. Após selecionadas, as ferramentas dissection tool e freehand sculpt tool foram utilizadas para iniciar o processo de dissecção, pois simulavam um bisturi, como é feito nos cadáveres formolizados. A ferramenta explore tool dialog foi empregada para identificar as estruturas anatômicas e nomear a visualização topográfica. Já a ferramenta pin model dialog disponibiliza alfinetes virtuais que servem para marcar órgãos e estruturas desejadas. Diferindo de imagens dimensionais, na mesa anatômica virtual $3 \mathrm{D}$ todas as estruturas selecionadas foram manuseadas, dissecadas, rotacionadas em diversos ângulos e redimensionadas. As imagens foram salvas por meio das ferramentas presets, snipping tool e property.

\section{Resultados}

A espécie felina foi selecionada no menu volume e as ferramentas dissection tool e freehand sculpt tool (Figura 1A) foram utilizadas para dar início ao processo de dissecção (Figuras 1B e C) dos sistemas digestório, urinário e cardiovascular (Figura 1D).

O sistema digestório foi explorado, sendo identificados: esôfago (Figura 2A), estômago, intestinos delgado e grosso. Foram realizadas anotações para identificar o corte anatômico. No software não havia detalhamento da cavidade oral. Todo o sistema esquelético do animal pôde ser visualizado (Figura 2B), demonstrando que o software também é eficiente para estudos anatômicos dos ossos, que são retratados na anatomia, uma vez que todos os dados da Anatomage são adquiridos por meio de exames de imagem.

No sistema urinário foram identificados e estudados: rins, ureteres, vesícula urinária e uretra (Figura 3A). As peças anatômicas virtuais mais detalhadas das estruturas de menor espessura, como os ureteres e a uretra, foram facilmente pesquisadas, confirmando que o estudo virtual oferece visualização mais clara em comparação às peças formolizadas. $O$ interior do rim pôde ser visualizado com o manejo da opacidade do tecido do órgão, outra funcionalidade da mesa anatômica virtual 3D. A região medular e o córtex dos rins, portanto, foram explorados com a ajuda dessa ferramenta, que facilita a visualização de estruturas internas de diversos órgãos (Figura 3B).

Os pulmões, a laringe e a traqueia foram estruturas evidenciadas no sistema respiratório (Figura 4 A e B). Entretanto, os brônquios, a porção nasal da laringe e a cavidade nasal não puderam ser exploradas, carecendo de detalhamento do software.

Durante a análise do sistema cardiovascular, somente o coração pôde ser estudado (Figura 5 A e B), uma vez que a funcionalidade de exibição vascular está disponível somente para a anatomia humana. A implementação do sistema vascular é possível com atualização do software. A inclusão deste seria de suma importância para o estudo anatômico, uma vez que é difícil estudar vascularização em cadáver formolizado, já que as estruturas são danificadas durante a preparação do cadáver. Na peça anatômica virtual humana é possível visualizar a vascularização completa do corpo, assim como o fluxo sanguíneo dos principais vasos e artérias. 

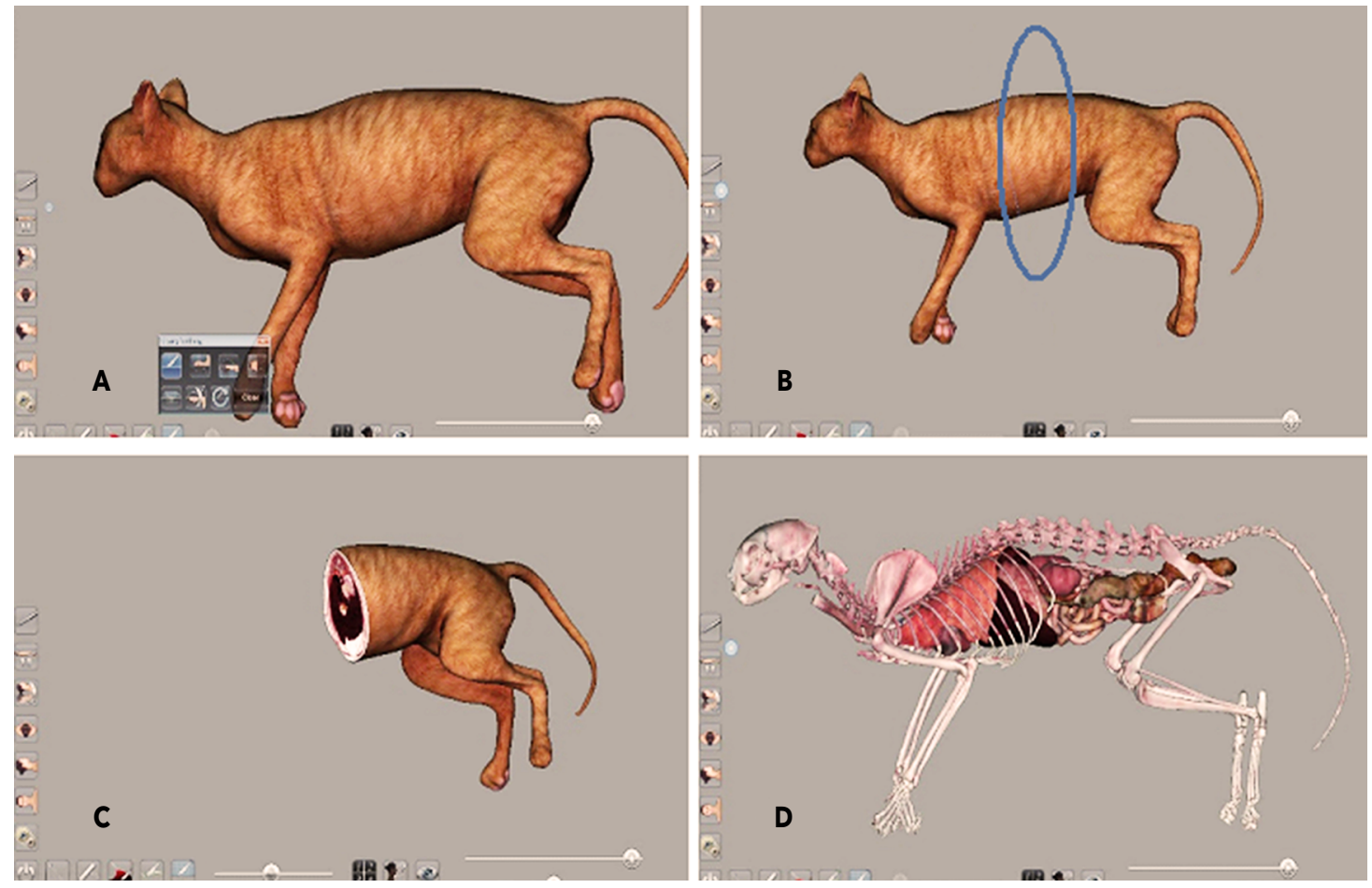

Figura 1 - Imagens 3D da espécie felina realizadas na Mesa Anatômica Virtual 3D (Anatomage). A) Vista lateral esquerda; utilização da ferramenta dissection tool. B) Vista lateral esquerda; círculo mostrando região da dissecção em corte transversal do tórax na mesa anatômica virtual. C) Vista lateral esquerda após a dissecção em corte transversal do tórax, utilizando a ferramenta dissection tool. D) Vista lateral esquerda, evidenciando os sistemas digestório, urinário, respiratório, cardiovascular e esquelético.
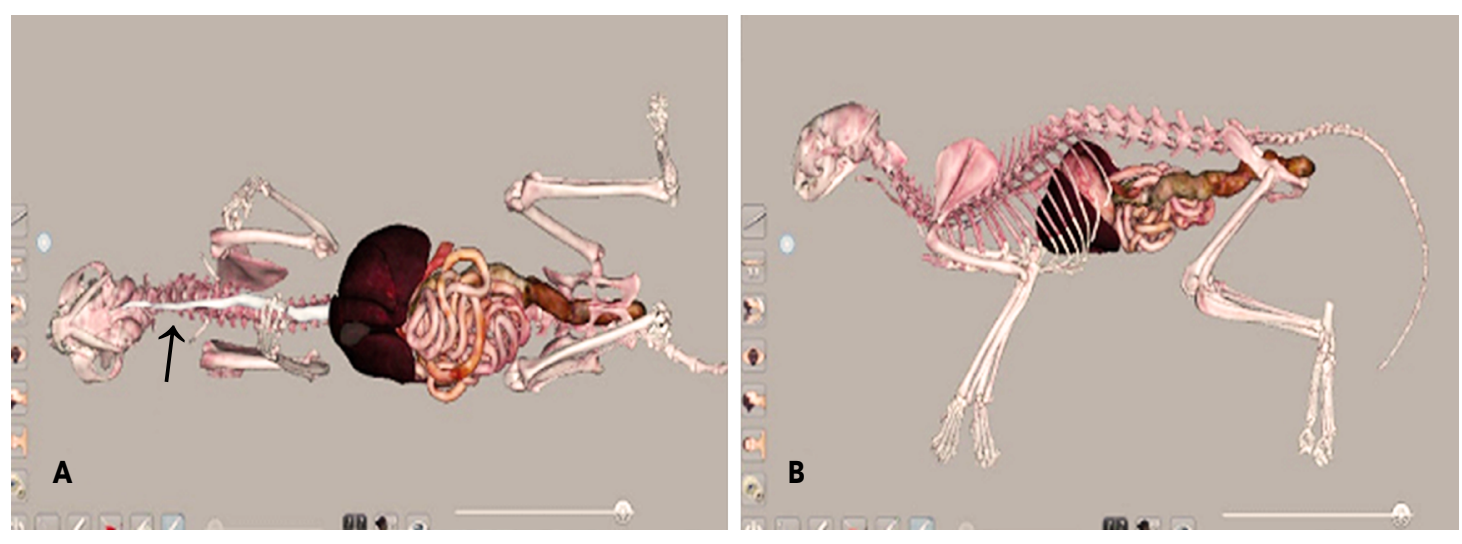

Figura 2 - Imagens 3D do sistema digestório da espécie felina, realizadas na Mesa Anatômica Virtual 3D (Anatomage). A) Vista dorsal, evidenciando o esôfago (SETA). B) Vista lateral esquerda; sistema digestório sem a presença do sistema respiratório. 

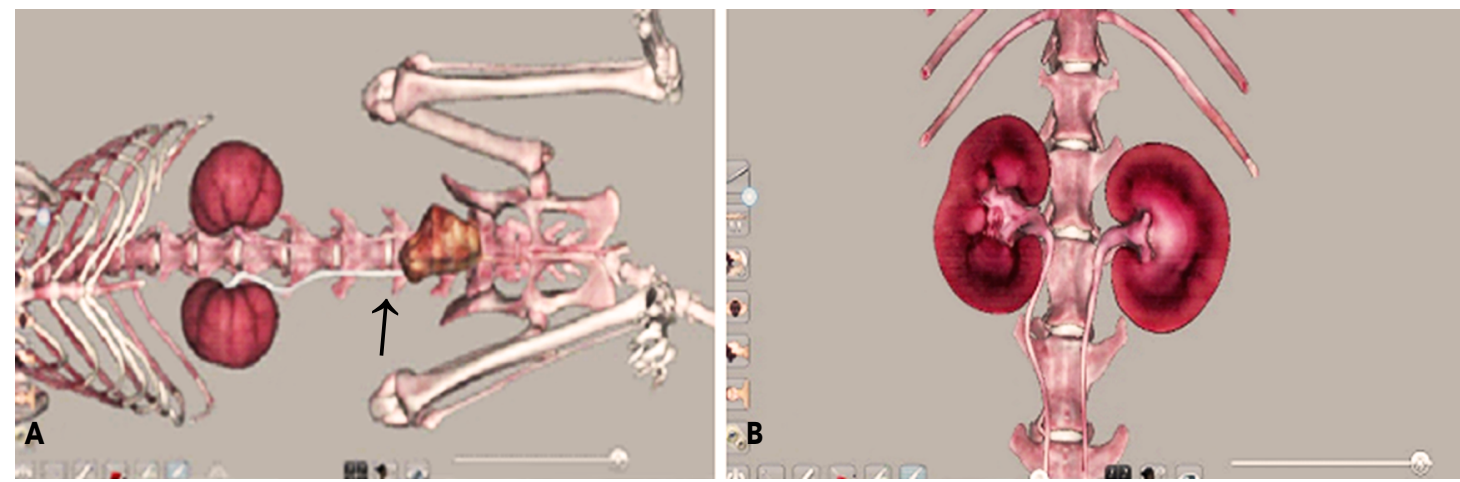

Figura 3 - Imagens 3D da espécie felina realizadas na Mesa Anatômica Virtual 3D (Anatomage). A) Visão dorsal, evidenciando o ureter esquerdo (SETA). B) Corte axial dos rins (vista dorsal).
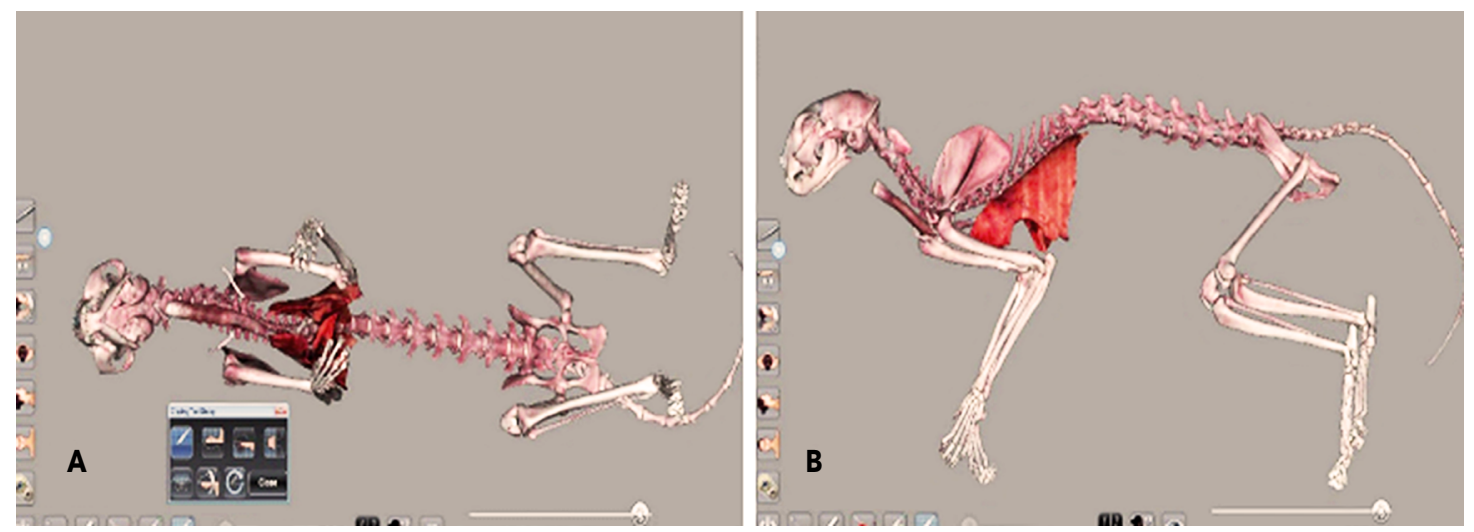

Figura 4 - Imagens 3D da espécie felina realizadas na Mesa Anatômica Virtual 3D (Anatomage). A) Vista ventral após o uso da ferramenta de dissecção no sistema respiratório. B) Vista lateral esquerda, evidenciando o pulmão.
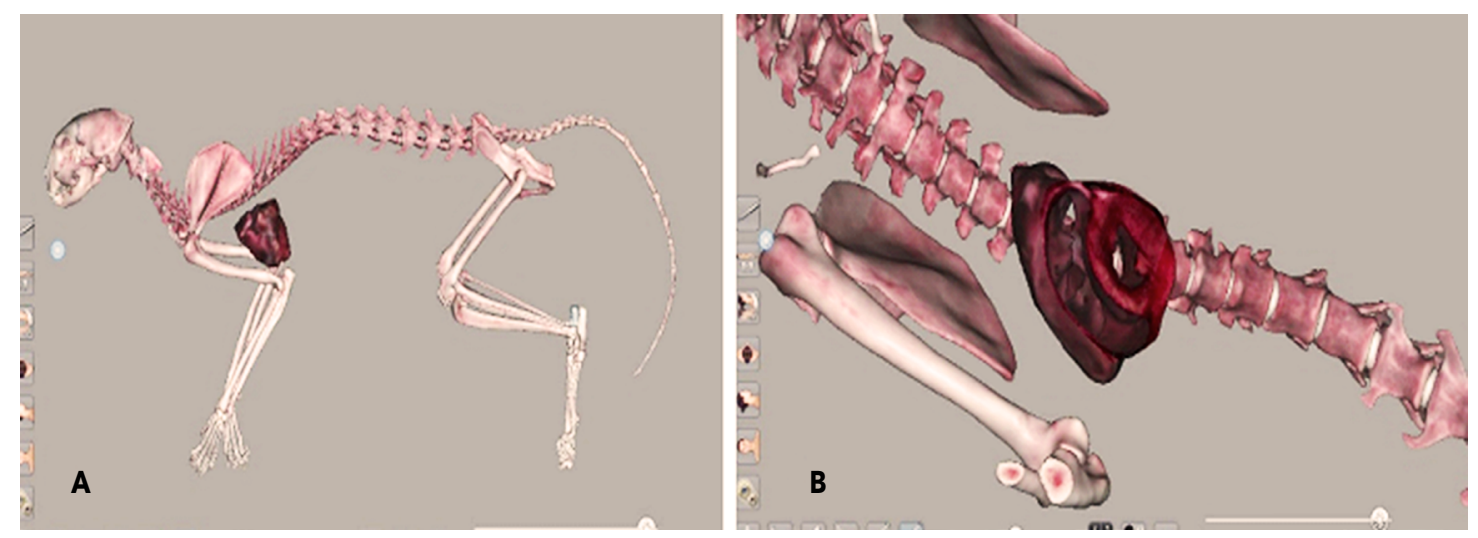

Figura 5 - Imagens 3D da espécie felina realizadas na Mesa Anatômica Virtual 3D (Anatomage). A) Vista lateral esquerda, evidenciando o sistema cardiovascular. B) Zoom na vista ventral, evidenciando (a parte interna). 
Durante o estudo anatômico virtual, os docentes relataram facilidade no manejo das ferramentas do software, por ser um sistema intuitivo e com design que proporciona simplicidade no seu uso, após capacitação. As ferramentas responsáveis pela denominação das estruturas possibilitaram que a identificação dos órgãos fosse feita de forma prática e sem necessidade de apoio literário. Por ser uma simulação virtual, não houve preocupação em danificar peças após o uso, pois qualquer procedimento aplicado pôde ser revertido e a estrutura retornou para seu estado inicial. Os docentes e discentes conseguiram treinar livremente cortes anatômicos com o bisturi virtual (dissection tool e freehand sculpt tool), propiciando autonomia nos estudos e ampliando habilidades práticas. Outro fator percebido foi o conforto de conduzir um estudo sem o odor do formol, utilizado para preparação das peças provenientes de cadáver.

Foi possível realizar anotações nas peças e salvar imagens dos cortes anatômicos, gerando material que docentes e discentes puderam consultar posteriormente acessando um pen drive. Em uma classe, o corpo discente é heterogêneo e cada indivíduo possui o próprio ritmo de aprendizado. Com a disponibilidade ilimitada de estruturas anatômicas e possibilidade de salvar imagens para estudo, a mesa anatômica mostrouse uma ferramenta flexível que se adequa ao ritmo único de cada aluno. Os participantes do projeto expressaram maior motivação para conduzir um estudo anatômico, sendo a Anatomage uma ferramenta facilitadora no ensino-aprendizagem. A praticidade da tecnologia foi e é um estímulo relevante para o meio acadêmico.

\section{Discussão}

É visível que a anatomia virtual simulada na Anatomage corresponde à anatomia real dos cadáveres formolizados. Custer e Michael (2015) e Chung et al. (2018) também pontuaram que as estruturas possuem detalhamento interno bem preciso na maioria dos sistemas, uma vez que os dados da mesa são adquiridos por meio de imagens obtidas por ressonância magnética, tomografia computadorizada e radiografias. Contudo, algumas estruturas poderiam ser aperfeiçoadas. Corrêa et al. (2019) pontuaram que a empresa Anatomage está disponível para atender as solicitações dos usuários da mesa. As atualizações no software poderiam sanar alguns aspectos como, por exemplo, integrar a funcionalidade de estudo vascular nas peças animais.

Diversos sistemas orgânicos podem ser explorados de forma eficaz utilizando a Anatomage, não apenas órgãos e tecidos moles, mas também sistemas locomotores, como as estruturas musculoesqueléticas. Os sistemas contemplados no presente estudo foram escolhidos aleatoriamente, tendo como foco a parte inicial da análise dessa ferramenta virtual como método complementar no ensino da anatomia na espécie felina. A pesquisa continua em desenvolvimento e o grupo pretende publicar outros estudos com os demais sistemas. Custer e Michael (2015), Gross e Masters (2017) e Periya e Moro (2019) corroboram este estudo ao comprovarem a eficácia desta tecnologia para estudo da anatomia. A presente pesquisa complementou a relevância do ensino de peças anatômicas virtuais com peças formolizadas.

Hisley et al. (2008) e Moraes et al. (2016) utilizaram o benefício de modificar o tecido estudado de acordo com a necessidade, alterando opacidade e tamanho. Igualmente, foi factível a alteração de opacidade da cápsula renal, possibilitando a exploração do interior do órgão, além do redimensionamento de estruturas menores, como ureter e uretra, que possuem pouca espessura. Em relação ao impacto da tecnologia 3D no meio acadêmico, a região estudada é um fator relevante, haja visto a dificuldade em estudar estruturas pequenas no cadáver formolizado (Raffan et al., 2017; Schoenfeld-Tacher et al., 2017; Corrêa et al., 2019). A tecnologia virtual é uma ferramenta excelente para combater a limitação de volume espacial encontrada na metodologia tradicional (Lisk et al., 2015; Raffan et al., 2017; Hisley et al., 2018; Corrêa et al., 2019), estando de acordo com os autores desse estudo.

O estudo virtual prova ser sensorialmente mais fácil para trabalhar, pois a ausência de formol torna o ambiente mais agradável para os discentes. 
Conforme Karam et al. (2016), existe uma relação entre o formol em peças anatômicas e danos à saúde humana. Ademais, as peças virtuais não deterioram com o passar do tempo e não são danificadas pelo manuseio excessivo. As peças formolizadas possuem vida útil menor, uma vez que o manuseio excessivo pode gerar danificações nas estruturas mais sensíveis (Fazan, 2011). Desta maneira, pesquisas com a tecnologia virtual podem ser realizadas sem preocupações quanto à durabilidade e delicadeza das estruturas.

Segundo Lisk et al. (2015) e Azer e Azer (2016), o design do software, disponibilidade visual da peça e interação cognitiva durante o uso do software são fatores que influenciam na eficácia de métodos virtuais, confirmando a percepção dos pesquisadores de que estes fatores agregam valor ao estudo. A facilidade de manejo do sistema, a disponibilidade tridimensional das peças em diversos planos (rostral, sagital, ventral e lateral) e o estímulo intelectivo gerado pelas funcionalidades da mesa foram fatores vantajosos durante esse estudo anatômico virtual.

Todavia, como apontado por diversos autores, foi perceptível a necessidade de integração da tecnologia 3D como método complementar à metodologia tradicional, o que trará benefícios para o meio acadêmico e melhorará o desempenho dos estudantes (Lisk et al., 2015; Raffan et al., 2017; Chung et al., 2018; Corrêa et al., 2019; SchoenfeldTacher et al., 2017). Pesquisadores como Santos et al. (2017b), Lima et al. (2018), Little et al. (2019) e Corrêa et al. (2019) afirmam que os docentes e discentes são favoráveis à nova tecnologia, a fim de amenizar dificuldades encontradas durante o estudo anatômico com peças formolizadas. Opiniões semelhantea foram observadas no presente trabalho.

\section{Conclusão}

Os recursos presentes no software certificaram que a Mesa Anatômica Virtual 3D (Anatomage) é uma ferramenta facilitadora, interativa e dinâmica. Após a capacitação da equipe, os sistemas digestório, urinário, respiratório e cardiovascular na espécie felina foram explorados, identificados e dissecados de forma clara e simples. É necessário atualizar a variabilidade das espécies animais no software, tendo em vista que alguns detalhes importantes de órgãos e sistemas não estão visíveis na ferramenta. A mesa anatômica virtual 3D apresenta um potencial que contribuirá de forma positiva na metodologia ativa, de forma a complementar o ensino tradicional de anatomia.

\section{Referências}

Azer SA, Azer S. 3D Anatomy models and impact on learning: a review of the quality of the literature. Health Prof Educ. 2016;2(2):80-98.

Brown J, Stonelake S, Anderson W, Abdulla M, Toms C, Farfus $A$, et al. Medical student perception of anatomage - A 3D interactive anatomy dissection table. Int J Surg. 2015;23(Suppl 1):S17-8.

Chung BS, Chung MS, Lee SB, Youn C, Park JS. Sectioned images of a cat head to contribute to learning of its sectional anatomy. Int J Morphol. 2018;36(2):537-43.

Cintra RB. Desafios do ensino da anatomia humana em faculdades de medicina. Rev Cient UMC. 2017;2(1):12.

Corrêa NF, Matos e Silva F, Cruz MC, Assumção RF, Leite DKVH. Mesa Anatômica virtual 3D: a tecnologia como um novo recurso didático na medicina veterinária. Encicl Biosf Centro Cient Conhecer. 2019;16(29):1044-54.

Custer TM, Michael K. The Utilization of the Anatomage Virtual Dissection Table in the education of imaging science students. J Tomogr Simul. 2015;1(1):102.

Fazan VPS. Métodos de ensino em anatomia: dissecção versus prossecção. O Antagonista: Rev de Divulgação Científica da Soc Bras de Anat. 2011;2(1):7-12.

Fontoura ELL, Mello ST, Gomes CRG, Mello JM. Conservação de peças anatômicas: vantagens e desvantagens de diferentes métodos. Rev UNINGÁ. 2020;57(2):34-46.

Fyfe S, Fyfe G, Dye D, Radley-Crabb H. The Anatomage table: differences in student ratings between initial implementation and established use. FoHPE. 2018;19(2): 41-52. 
Gross M, Masters C. Virtual dissection: using active learning with the anatomage table to enhance student learning. FASEB J. 2017;31(S1):lb9.

Gummery E, Cobb KA, Mossop LH, Cobb MA. Student perception of veterinary anatomy practical classes: a longitudinal study. J Vet Med Educ. 2018;45(2):163-76.

Hisley KC, Anderson LD, Smith SE, Kavic SM, Tracy JK. Coupled physical and digital cadaver dissection followed by a visual test protocol provides insights into the nature of anatomical knowledge and its evaluation. Anat Sci Educ. 2008;1(1):27-40.

Karam RG, Cury FS, Ambrósio CE, Mançanares CAF. Uso da glicerina para substituição do formaldeído na conservação de peças anatômicas. Pesq Vet Bras. 2016;36(7):671-5.

Lima FT, Sturn RM, Ribeiro ARB. Uso de animais no ensino da medicina veterinária: métodos substitutivos. Nucleus. 2018;15(2):251-64

Lisk K, McKee P, Baskwill A, Agur AMR. Student perceptions and effectiveness of an innovative learning tool: anatomy glove learning system. Anat Sci Educ. 2015;8(2):140-8.

Little WB, Artemiou E, Fuentealba C, Conan A, Sparks C. Veterinary students and faculty partner in developing a virtual three - Dimensional (3D) interactive touch screen canine anatomy table. Med Sci Educ. 2019;29:223-31.

Massari CHAL, Schoenau LSF, Cereta AD, Miglino MA. Tendências do ensino de anatomia animal na graduação de medicina veterinária. Rev Grad USP. 2018;3(2):25-32.

Moraes GNB, Schingel PA, Silva Jr EX. Uso de roteiros didáticos e modelos anatômicos, alternativos, no ensinoaprendizagem nas aulas práticas de anatomia humana. Rev Ibero-Am Estud Educ. 2016;11(1):223-30.

Periya SN, Moro C. Applied learning of anatomy and physiology: virtual dissection tables within medical and health sciences education. BKK Med J. 2019;15(1): 121-7.
Raffan H, Guevar J, Poyade M, Rea PM. Canine neuroanatomy: Development of a 3D reconstruction and interactive application for undergraduate veterinary education, PloS One. $2017 ; 12(2)$ : e0168911.

Reis C, Martins MM, Mendes RAF, Gonçalves LB, Sampaio Filho HC, Morais MR, et al. Avaliação da percepção de discentes do curso médico a cerca do estudo anatômico. Rev Bras Educ Med. 2013;37(3):350-8.

Reis DAL, Gouveia BLR, Alcântara BM, Saragiotto BP, Baumel EED, Ferreira JS, et al. Biomodelos ósseos produzidos por intermédio da impressão 3d: uma alternativa metodológica no ensino da anatomia veterinária. Rev Grad USP. 2017;2(3):47-53.

Rinaldi VD, Lorr NA, Williams K. Evaluating technology supported interactive response system during the laboratory section of a histology course. Anat Sci Educ. 2017;10(4):328-38.

Salbego C, Oliveira EMD, Silva MAR, Bugança PR. Percepções acadêmicas sobre o ensino e a aprendizagem em anatomia humana. Rev Bras Educ Med. 2015;39(1):23-31.

Santos AA, Silva MGV, Santos JL, Melo PGB. Principais métodos de fixação de peças para estudo anatômico: uma revisão de literatura. Arq Mudi. 2017a;21(1):19-25.

Santos SLF, Alves HHS, Saraiva HSTT, Barros KBNT. Ferramentas tecnológicas aplicadas ao processo ensinoaprendizagem em anatomia humana. Rev Educ Meio Amb Sau. 2017b;7(4):64-72.

Schoenfeld-Tacher RM, Horn TJ, Scheviak TA, Royal KD, Hudson LC. Evaluation of $3 D$ additively manufactured canine brain models for teaching veterinary neuro-anatomy. J Vet Med Educ. 2017;44(4):612-9.

Silva CF, Ribeiro FS, Reges ACF, Azevedo RTS, Silva Jr EX, Cerqueira GS, et al. Análise de satisfação do uso de exames de imagens, como proposta complementar, no processo de ensino e aprendizagem da anatomia sistêmica. Rev Saude Cienc. 2018;7(1):35-44 